Kardiologe 2017 · 11:332-335

DOI 10.1007/s12181-017-0183-8

Online publiziert: 4. August 2017

C) Springer Medizin Verlag GmbH 2017

CrossMark

Liebe Leserinnen und Leser,

das deutsche Gesundheitswesen hat in den letzten 40 Jahren zahllose Belastungsproben erleben müssen - und auch überstanden. Wenn auch darüber gestritten werden mag, ob gut oder schlecht. In jedem Fall existiert es noch als wesentlicher Bestandteil der Solidargemeinschaft und gilt mit Recht als eines der besten wenn nicht das beste - Gesundheitssystem der Welt: Nirgendwo sonst haben so viele Menschen so zeitnahen und uneingeschränkten Zugang zu einer derart hochwertigen und flächendeckenden medizinischen Versorgung.

Möglicherweise liegt es in der Natur von Menschen und Problemen, dass einem die aktuellen Umbrüche und möglicherweise drohende Gefahren immer als die Dramatischsten erscheinen, denen man jemals ausgesetzt war. In jedem Fall ist das Gesundheitswesen aktuell in einer Phase, in der man zu der Einschätzung kommen kann, dass die aufgetretenen Verwerfungen und anstehenden Probleme ein bisher nie gekanntes Ausmaß erreicht haben (• Abb. 1). Dies gilt dabei sowohl mit Blick auf die Patienten wie auch die Ärztinnen und Ärzte (heutzutage gerne nüchtern als „Leistungserbringer“ bezeichnet) und auch alle anderen damit befassten Personen wie aber auch die das Gesundheitssystem tragenden Organisationen. Immer mehr alte und auch krankere Patienten, die auch steigende Er-

Prof. Dr. Holger Reinecke ist Vorsitzender des Ständigen Ausschusses "Leistungsbewertung in der Kardiologie - Ausschuss DRGs" der Deutschen Gesellschaft für Kardiologie und Vorsitzender des Kuratoriums für Fragen der Klassifikation im Gesundheitswesen beim Bundesministerium für Gesundheit.

\title{
H. Reinecke
}

Department für Kardiologie und Angiologie, Universitätsklinikum Münster, Albert-Schweitzer-Campus 1, Münster, Deutschland

\section{Ökonomie und Ethik in der Medizin}

wartungen an ihre Gesundheit und Mobilität haben, begrenzte Ressourcen mit einer sich ausbreitenden, geradezu paradox erscheinenden Maxime zur „Gewinnerzielung“ bei der Versorgung kranker Menschen (warum sollte man bei der Versorgung Kranker überhaupt einen Gewinn erzielen?), teils erheblich verfallende Vergütungen, ausufernde medizinische Leistungen mit teils fraglicher Indikation, dramatische Probleme mit dem Nachwuchs in allen Berufsgruppen - dies alles entwickelt sich bedrohlich. Diese Entwicklungen verändern die medizinische Versorgung und ganz unmittelbar die tägliche Betreuung der Patienten so eingreifend, dass es dringlich geboten erscheint, sich auf die fundamentalen Werte $\mathrm{zu}$ besinnen und das eigentliche Ziel aller Beteiligten und auch allen ärztlichen Handelns - das Wohl des Patienten und die Fürsorge für ihn - wieder gemeinschaftlich in den Vordergrund zu stellen. Dass dabei auch eine implizite Verantwortung besteht, die von der Solidargemeinschaft zur Verfügung gestellten Ressourcen verantwortungsvoll und effektiv einzusetzen, ist selbstverständlich.

\section{Gesundheitspolitische Aktivitäten der DGK}

Die Deutsche Gesellschaft für Kardiologie (DGK) wurde als medizinischwissenschaftliche Fachgesellschaft gegründet und musste sich jedoch zur Wahrung dieses Primats in den letzten Jahrzehnten immer mehr auch mit politischen und gesundheitsökonomischen Aspekten beschäftigen. Nur so konnte und kann sichergestellt werden, dass die DGK ihre originären Funktionen ausüben und ihre Mitglieder und me- dizinisch-wissenschaftlichen Interessen angemessen vertreten kann. So wurden in den letzten Jahren zahlreiche, mehr und mehr auch gesundheitspolitisch tätige Gremien und Ausschüsse innerhalb der DGK etabliert. Eines dieser Gremien ist der ständige Ausschuss „Leistungsbewertung in der Kardiologie - Ausschuss DRGs“, der sich mit dem Fallpauschalen-basierten Abrechnungssystem in Deutschland und dem damit zusammenhängenden Themenkomplex und den zugehörigen Klassifikationssystemen beschäftigt. Vom Vorstand der Deutschen Gesellschaft für Kardiologie schon sehr früh im Jahr 2002 eingerichtet, war es aber auch immer ein Anliegen dieses früher als "Projektgruppe DRGs“ bezeichneten Gremiums, auch über nüchtern-rationale Aspekte von Klassifikationen und Gruppierungssysteme hinaus zu schauen auf perspektivische, aber auch ethische Entwicklungen.

\section{I) Fortschritt ist ohne Veränderung unmöglich; und wer seine Denkweise nicht verändern kann, kann gar nichts verändern. (George Bernard Shaw)}

In diesem Rahmen war es erfreulicherweise auf der diesjährigen Frühjahrstagung der DGK in Mannheim im April 2017 möglich, dass eine von den Professoren Yskert von Kodolitsch (Hamburg), Jochen Reinöhl (Freiburg/Bad Krotzingen) und Lutz Frankenstein (Heidelberg) organisierte Sitzung zu perspektivischen und ethischen Themen stattfinden konnte. Drei exzellente Redner haben in 
Hier steht eine Anzeige.

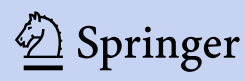




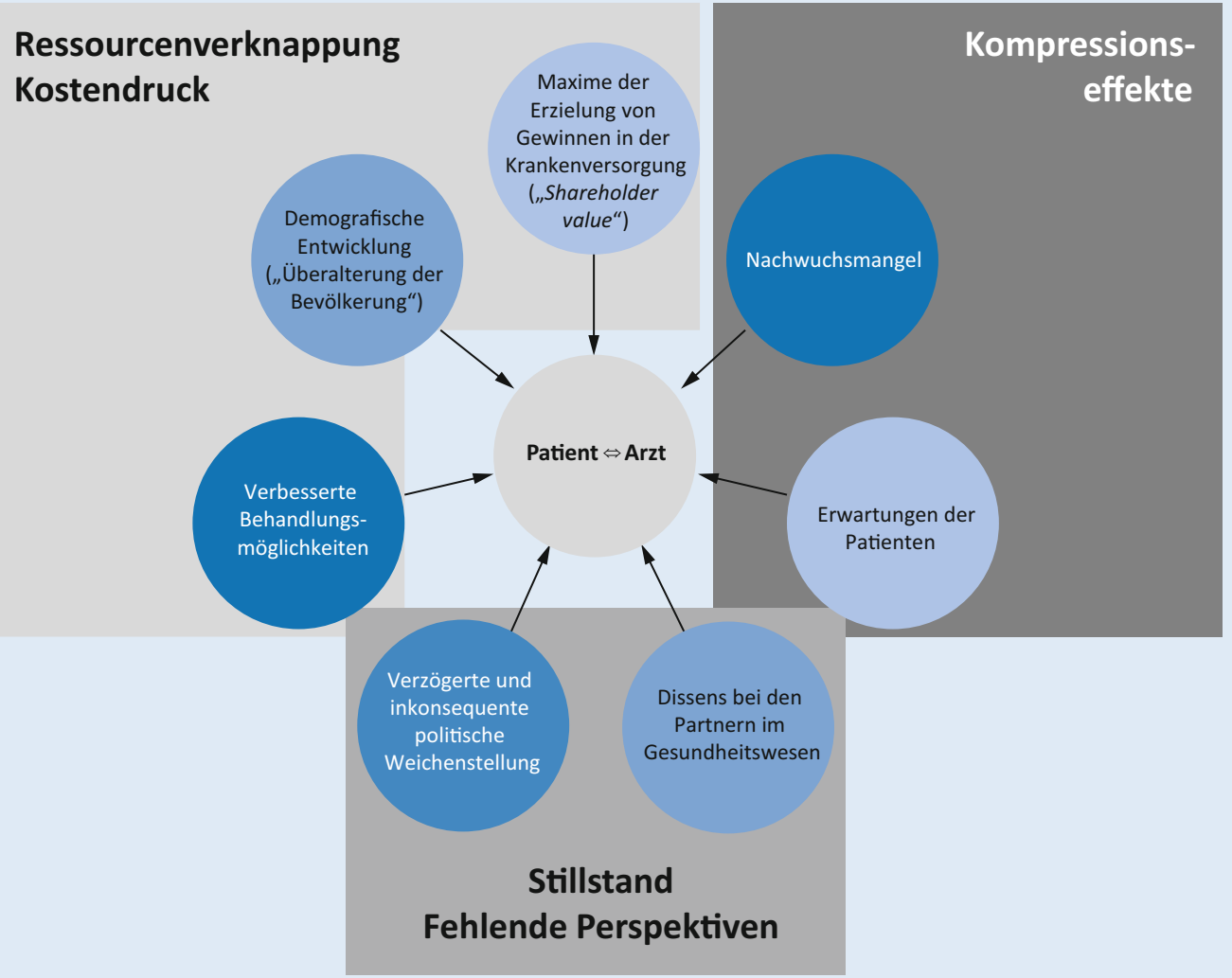

Abb. $1 \triangleleft$ Einflussgrößen auf das Arzt-Patienten-Verhältnis

\section{Infobox Arzt-Patienten-Verhältnis}

Die - Abb. 1 zeigt einen Auszug von zahlreichen Einflussgrößen, die zunehmend das Arzt-Patienten-Verhältnis belasten (oder wie es eigentlich korrekterweise - mit Blick auf den wichtigeren Partner - heißen sollte: das Patient-Arzt-Verhältnis). Obwohl es auf den ersten - aber auch jeden weiteren - Blick völlig abwegig erscheint, mit der selbstlosen und nur als Zuwendung verstehbaren Behandlung von kranken Menschen Gewinne erzielen zu sollen bzw. sogar zu müssen, ist dies als Maxime in den letzten Jahrzehnten immer mehr in den Vordergrund gestellt worden. Ganze Gesundheitskonzerne erwirtschaften teils hohe Gewinne, die sie an ihre Besitzer und Aktieninhaber weiterreichen - und damit dieses Geld dem Gesundheitssystem entziehen. Weitere Entwicklungen demografischer Art (z. B. die Überalterung der Bevölkerung, aber auch das Verhältnis von Beitragszahlern und Nichtzahlern) wie aber auch die an sich sehr erfreulichen, deutlich verbesserten medizinischen Behandlungsmöglichkeiten, die natürlich ihren Preis haben, können zusammengefasst werden als Ressourcenverknappung und erhöhter Kostendruck. Demgegenüber steht bei den versorgenden Ärztinnen und Ärzten ein stärker werdender Nachwuchsmangel.Zudem gibt es auch eine deutliche Erhöhung der Erwartungen der Patienten, die zweifellos (zumindest größtenteils) berechtigt ist: Jeder hat den berechtigten Wunsch, so lange zu leben, wie es geht, und das auch möglichst mit einer nicht bzw. nur gering eingeschränkten Lebensqualität. Diese verstärkten Erwartungen in Kombination mit dem Nachwuchsmangel setzen aber die im System tätigen Ärztinnen und Ärzte - aber auch zweifellos die Pflege und andere Berufsgruppen - unter einen immer größeren Druck, der als Kompressionseffekt beschrieben werden kann: Immer mehr Leistung muss von (zumindest relativ gesehen) immer weniger Menschen erbracht werden.

In dieser schwierigen Situation verschlechtert ein nach Meinung des Verfassers bedauerlicherweise um sich greifender Dissens bei den Partnern im Gesundheitswesen die Handlungsfähigkeit. Gleichzeitig erfolgt eine politische Weichenstellung verzögert bzw. inkonsequent und ist daher nicht in der Lage, eine durchgreifende Verbesserung der Situation zu erreichen. Dieser Komplex des Stillstands und der fehlenden Perspektiven blockiert damit jeglichen schnellen Ausweg aus der aktuell immer schwieriger werdenden Lage. diesem Spannungsfeld von Ökonomie und Ethik herausragende Vorträge gehalten haben. Diese Gedanken möchten wir durch diesen Themenschwerpunkt einer größeren Zahl von Kolleginnen und Kollegen zugänglich machen. So abwechslungsreich wie die Inhalte und Zielsetzungen dieser Vorträge sind dabei auch die verwendeten stilistischen Mittel der Vortragenden. Teil geschieht dies sachlich-analytisch, teils philosophisch und teilweise auch in durchaus provokanter Art und Weise. Dies erscheint der Komplexität der Themen wie auch der derzeit sich immer mehr abzeichnenden Stase bei der eigentlich dringlich benötigten Lösung drängender Probleme durchaus angemessen. Gleichgültig, ob man sich nun darin ganz oder teilweise wiederfinden kann oder mehr oder weniger deutlich davon distanzieren möchte: Wir müssen uns intensiv damit auseinandersetzen.

\section{Quo vadit?}

Es war das Ziel der Initiatoren der Sitzung in Mannheim, eine über den Kongress 
hinausgehende Auseinandersetzung mit diesen Problemen für jeden Einzelnen, die Fachgesellschaft und auch die Institutionen, wie z.B. die Krankenhäuser, in denen wir tätig sind, zu erreichen. Auch wenn zahlreiche der aktuellen Entwicklungen im Gesundheitswesen sich als hoch problematisch darstellen und eine Lösung kaum bzw. nicht absehbar erscheint, plädieren letztlich alle Vortragenden inklusive des Verfassers dieser Einleitung dafür, sich die Initiative - das sog. „Heft des Handelns“ - nicht aus der Hand nehmen zu lassen. Dies ist zweifellos mühsam und langwierig. Eine pessimistisch zurückhaltende oder gar defätistische Einstellung birgt aber die Gefahr, dass allein andere die wesentlichen Entscheidungen treffen. Insofern sind unser aller Aktivitäten alternativlos, gleichgültig wie mühselig sie sein mögen.

Dabei erscheint das bisher Erreichte mit Blick auf die letzten Jahrzehnte der Aktivität der Fachgesellschaft DGK und auch der 15 Jahre Arbeit der Projektgruppe durchaus als erfolgreich und nachhaltig. Besonders herausgestellt werden sollten hier zahlreiche, erfolgreiche konsentierte Aktivitäten mit anderen Fachgesellschaften die in fruchtbare politische Initiativen gemündet sind. Aber auch sehr hilfreiche Konsensusvereinbarungen mit den Kolleginnen und Kollegen des Medizinischen Dienstes der Krankenkassen (MDK) und des Spitzenverbandes der Gesetzlichen Krankversicherungen (GKV) belegen, dass gute Kompromisse möglich sind und für alle Seiten deutliche Fortschritte und Verbesserungen bringen $[1,2]$.

\section{》) Unsere größte Schwäche liegt im Aufgeben. Der sichere Weg zum Erfolg ist immer, es doch noch einmal zu versuchen. (Thomas A. Edison)}

Letztendlich kann daher am Ende nur die Schlussfolgerung stehen, dass medizinische Versorgung im Alltag aber auch im "großen Gesundheitswesen“ nicht allein von Betriebswirtschaftlern, Politikern oder Juristen gemacht werden kann. Dies ist schlichtweg faktisch nicht möglich und der Versuch nicht sinnvoll. Deren Kompetenzen sind wichtig und hilfreich, aber sie ersetzen nicht die Nähe zum Patienten und das Antizipieren seiner Bedürfnisse. Den Kurs bestimmen sollten diejenigen, die die medizinische Versorgung und die Verantwortung beurteilen und auch letztendlich jeden Tag tragen, egal, ob sie nun unmittelbar die Patienten betreuen oder indirekt bei den im Gesundheitswesen tätigen Institutionen arbeiten: wir Ärztinnen und Ärzte.

Ihr

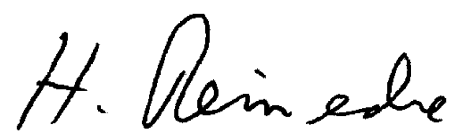

Prof. Dr. Holger Reinecke

\section{Korrespondenzadresse}

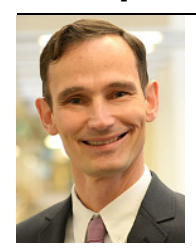

Prof. Dr. H. Reinecke

Department für Kardiologie und Angiologie, Universitätsklinikum Münster, Albert-

Schweitzer-Campus 1

Gebäude A1, 48149 Münster, Deutschland

Holger.Reinecke@

ukmuenster.de

Interessenkonflikt. H. Reinecke gibt an, dass er Vortragshonorare von BMS, MedUpdate, NephroUpdate und Pfizer erhielt. Er agiert als Berater für BMS, Pfizer und Pluristem. Die von ihm geleitete Abteilung erhält Vergütungen für die Teilnahme an Multicenterstudien von Bard, Bayer, Biotronik und Pluristem.

\section{Literatur}

1. Reinecke $H$, Braun M, Frankenstein L et al (2015) Kriterien für die Notwendigkeit und Dauer von Krankenhausbehandlung bei Koronarangiografien und -interventionen. Kardiologe 9:295-302

2. Schumacher Betal (2017) Kriterien für die Notwendigkeit und Dauer von Krankenhausbehandlung bei elektiven rhythmologischen Eingriffen. Kardiologe 11:209-218
Auch Online-Zugang zu allen Beiträgen Ihres Zeitschriftenabonnements

\section{Wussten Sie, dass Sie als Abonnent dieser Zeitschrift automatisch Online-Zugriffsrechte auf das gesam- te Beitragsarchiv und die CME-Kurse haben?}

Der Zugang zu Ihrer Online-Bibliothek und den CME-Kursen ist im Abonnement Ihrer Zeitschrift inbegriffen. Für den Zugang müssen Sie sich lediglich einmal über www.springermedizin.de/register registrieren.

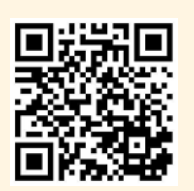

Über diesen QR-Code schnell und einfach registrieren

Bitte nutzen Sie für die Registrierung Vorund Nachname und Lieferadresse wie beim Abonnement der Zeitschrift (s. Adressaufkleber auf Ihrem Heft). So kann im System die Zugehörigkeit zu Ihrer Zeitschrift sichergestellt werden.

Aufgrund des Heilmittelwerbegesetzes dürfen die Inhalte der Website nur medizinischen Fachkreisen zur Verfügung gestellt werden. Bei der Anmeldung bitten wir Sie deshalb, einen Berufsnachweis vorzulegen.

Bei Medizinern mit Mitgliedschaft in der deutschen Ärztekammer reicht die einheitliche Fortbildungsnummer

(EFN). Alternativ schicken Sie eine Bestätigung des Arbeitgebers, Studiennachweis oder andere Zeugnisse ganz unkompliziert an kundenservice@springermedizin.de.

Mit Benutzername und Passwort haben Sie außerdem Zugang zu den freien Inhalten auf den Seiten von: www.springermedizin.de www.aerztezeitung.de

Sollten Fragen oder Probleme auftauchen, wenden Sie sich einfach an Ihren Kundenservice:

kundenservice@springermedizin.de 